\title{
Current Research on Biomedicine
}

\section{Emanual Michael Patelia*}

Department of Pharmacology, University of Bedfordshire, UK

\begin{abstract}
An annual sum of approximately $£ 2.8$ billion is spent on academic medical research in the UK $-£ 1.2$ billion of which is sourced from medical charities. Despite only being a fraction of the amount spent on medical research in the US, the UK is second only to the US in terms of research output, with more articles and citations per researcher than any other country. But how much research with a medical aim is actually converted into a benefit for patients? Is it all money well spent? These are the questions being asked as we endure an economic downturn, while patients seek the next miracle cure.
\end{abstract}

\section{Introduction}

Are scientists doing enough to translate their findings to the clinic? When it comes to investigating a medical problem, the mindset of a scientist can be somewhat different to what a layperson might expect. They are often driven by their fascination in the mechanisms of disease. But often their sources of funding are either the government or charities relying on the generosity of members of the public $[1,2]$. These sources award funding to researchers with the hope that they will make the next discovery or innovation to improve prevention, diagnosis or treatment of disease and ultimately save lives. One could, however, argue that extensive work is necessary to fully understand the problem before any steps towards translational research can be made. So are scientists doing enough to translate their findings to the clinic? And hence do they justify the funding they receive?

Perhaps herein lies a problem with the way scientific research is recognized. Scientists build their reputation on a myriad of publications, ideally in high impact factor journals, and the ability to attract large grants. Hence we often see publication of a research paper as the end point of a research project. The peer-review process is important to judge the quality of the study and whether its results and conclusions are reliable. Thus it is necessary to ensure the safety and efficacy of any potential therapeutics in question. Unfortunately, at times the peerreview system can be flawed, as journal editors prioritise studies with positive results, particularly on so-called "trendy topics", such as the use of stem cells in regenerative medicine. This means negative data is often ignored even though it could be highly informative on the efficacy of a particular treatment or understanding the pathogenesis of disease. Hence researchers choose not to submit a paper showing negative data as they are resigned to believe it will never be accepted for publication. While initiatives such as the Journal for Negative Results in Biomedicine [3] aim to counteract this problem, they have yet to gain widespread recognition.

This sets a dangerous precedent for many reasons. Several groups may come up with the same idea that a particular protein may be detrimental in cancer for example. However if a group has already carried out a study, which showed no effect, the rest of the scientific community will never know. Thus other groups will waste time and resources on a study, which has already been performed, possibly many times over. What is of great concern is that therapies that have no beneficial effect in treating disease may still be produced and given to patients because studies showing a beneficial effect have been published while studies showing no benefit have not. Worryingly, recent surveys show that at least $50 \%$ of published research may not be reproducible. Ben Goldacre brilliantly explains the publication bias of clinical trials in his recent TedMed talk [4]. Publication bias has been extensively studied with regard to clinical trials but less attention has been paid to basic scientific studies, which experience the same kind of discrimination and whose results are the first step in producing potential therapies.

But with success in publishing comes a greater chance of success with grant applications, as having a good track record proves your ability to produce high quality results. Recently there has been a developing trend towards awarding larger grants to more prominent scientists over a longer period of time in an effort to inspire greater discoveries. But could such an initiative change scientists' motivation towards obtaining larger amounts of funding rather than producing sound science that is ultimately beneficial to the public? A recent study in Canada suggests researchers receiving additional funding were not more productive [5]. Instead it appears that awarding smaller grants to more researchers boosts productivity.

The inability to translate biomedical research findings could be attributed to the increasing divide between researchers and clinicians, which is a relatively recent phenomenon. While clinicians performed early research, the emergence of molecular biology 40 years ago led to specialized research by biomedical scientists, who have greatly increased the understanding of disease in recent decades, but it appears few have meaningful collaborations with clinicians or industry. This is possibly due to reluctance among basic scientists to delve into the clinical situation while clinicians, whose time is occupied with patients, have difficulty not only performing research but also just keeping in touch with the latest literature, which is increasingly complex.

Fortunately this problem is being realised by more individuals in the scientific community. Funding organisations are making a concerted effort to encourage multi-disciplinary research by enforcing collaborations between biomedical scientists, engineers and clinicians. The National Institutes of Health (NIH) in the US have created a translational research initiative, pumping funding into the creation

*Corresponding author: Emanual Michael Patelia, Department of Pharmacology, University of Bedfordshire, UK, E-mail: ricky.emanual@gmail.com

Received August 22, 2013; Accepted September 22, 2013; Published September 26, 2013

Citation: Patelia EM (2013) Current Research on Biomedicine. J Bioanal Biomed 5: 097-098. doi:10.4172/1948-593X.1000087

Copyright: (c) 2013 Patelia EM. This is an open-access article distributed unde the terms of the Creative Commons Attribution License, which permits unrestricted use, distribution, and reproduction in any medium, provided the original author and source are credited. 
of numerous Clinical and Translational Science Centers (CTSCs) across the country. These research centers are at the early stages of their development in many cases but have still yielded some positive signs thus far. Much of the research conducted in CTSCs is driven towards drug development and there have been many drug targets identified which have led to drug development and even the initiation of clinical trials for example against cancer, neurological diseases, and cardiac disorders (through the advancement of regenerative medicine). However it is not clear what proportion of research within CTSCs has been successful in achieving a translational output.

Similar efforts have been seen in the UK with the creation of specialist research centers such as the British Heart Foundation Centers of Research Excellence and MRC (Medical Research Council) UK Centre in Allergic Mechanisms of Asthma. These facilities provide scientists with state-of-the-art facilities, renewed sources of funding and a stronger platform to foster multi-disciplinary collaborations. There has been a strong initiative to bring academics closer to the clinical setting with the creation of the National Institute of Health Research (NIHR), acting as a bridge between basic research and the delivery of improvements to the clinic. The NIHR has several aims: funding research for the benefit of patients, such as public health research or the development of innovative medical technologies; increasing the reliability and open access to medical research literature to better inform patients, clinicians, and policy-makers in their decision making with regards to medical practice; and improving the healthcare infrastructure. Various teams have also been set up to facilitate partnerships with industry to develop pharmaceuticals, medical technologies, and improvements in the healthcare environment.

\section{Summary}

It is evident that the increasing need to translate basic scientific research to the clinical setting is being recognised by funding agencies and national healthcare institutions. The increase in funding and provision of modern research facilities is encouraging for the future of medicine. Scientific developments over the next decade will be the true testament of the success of current translational research programmes.

\section{References}

1. http://www.amrc.org.uk/home/

2. https://www.gov.uk/government/uploads/system/uploads/attachment_data/ file/32489/11-p123-international-comparative-performance-uk-researchbase-2011.pdf

3. Journal of Negative Results in Biomedicine

4. http://www.ted.com/talks/ben_goldacre_what_doctors_don_t_know_about_ the_drugs_they_prescribe.html

5. Michel JF, David JC (2013) Big Science vs. Little Science: How Scientific Impact Scales with Funding. PLOS One 8: e65263. 